\title{
Refugios diurnos del murciélago Rhynchonycteris naso (Chiroptera: Emballonuridae) en Laguna de Términos, Campeche, México
}

\author{
Karla P. Borges-Jesús ${ }^{1,2 *}$, José D. Cú-Vizcarra ${ }^{3,4}$, Griselda Escalona-Segura ${ }^{4}$
}

\& Jorge A. Vargas-Contreras ${ }^{1}$

1. Facultad de Ciencias Químico Biológicas, Universidad Autónoma de Campeche, Avenida Exhacienda Kalá, San Francisco de Campeche, Campeche, 24085, México; karlaborges83@gmail.com (*Correspondencia), javargas@uacam.mx

2. Red de Biología y Conservación de Vertebrados, Instituto de Ecología, A.C., Carretera Antigua a Coatepec 351, El Haya, Xalapa, Veracruz, 91073, México.

3. División de Posgrado, Instituto de Ecología, A.C., Carretera Antigua a Coatepec 351, El Haya, Xalapa, Veracruz, 91073, México; jcvizcarra104@gmail.com

4. Departamento de Conservación de la Biodiversidad, El Colegio de la Frontera Sur, Avenida Rancho Polígono 2-A, Ciudad Industrial, Lerma, Campeche, 24500, México; gescalon@ecosur.mx

Recibido 25-VIII-2020. Corregido 26-XI-2020. Aceptado 02-XII-2020.

\begin{abstract}
Diurnal roosts of the bat Rhynchonycteris naso (Chiroptera: Emballonuridae) in Laguna de Términos, Campeche, Mexico. Introduction: The selection of day-roost is a crucial process for bats because it influences the ecology, social organization, and survivorship aspects of their populations. Proboscis bat (Rhynchonycteris naso) is a protected species by Mexican law, whose foraging and roosting areas are strongly associated with bodies of water, primarily rivers. Therefore, alterations in the water quality pose a risk to their populations. However, knowledge of the current state of their populations and roosting ecology is very poor in Mexico. Objectives: To locate and to describe $R$. naso's diurnal roosts, and to evaluate the relationship of the group size per roost with the characteristics of the diurnal roost, the salinity and $\mathrm{pH}$ of the water where they inhabit. Methods: From March to October 2015, we searched diurnal roost through boat tours along rivers, streams, and lagoons, in eleven sites in the Laguna de Términos Flora and Fauna Protection Area, Campeche, Mexico. For each localized roost, we recorded the group size, and their spatial distribution, the type of roost and vegetation, the distance between roosts, the diameter of trunk, the salinity, and $\mathrm{pH}$. In order to investigate the effect of the variables recorded in diurnal roost on size group, generalized linear models were adjusted. Results: We found 48 diurnal roost in dead tree-trunks, tree bark and bridges, associated with freshwater, and oligohaline bodies, mainly in mangrove vegetation. The average size of the groups was $5.3 \pm 0.48$ and $22.6 \pm 9.54$ in natural and artificial roosts, respectively. We found a significant relationship with the type of roost, type of vegetation and diameter of trunk. Roosts with larger groups of bats presented an aggregated distribution. We located 40 diurnal roosts on freshwater bodies and eight in oligohaline with a high grouping level. Conclusions: Greater incidence of roost in fresh and lightly acid water is explained under optimal foraging theory. Because the study area is found in the limit of their distribution, mangrove is a very important vegetation type to guarantee the establishment of $R$. naso populations. Also, due no roosts were found in some portions of the study zone, quality of water, and contaminant level studies are crucial. This study provides relevant information on their populations with direct implications for the conservation in coastal areas.
\end{abstract}

Key words: perch site; physicochemical parameters; proboscis bat; protected area; roosting ecology; salinity; threatened species.

Borges-Jesús, K.P., Cú-Vizcarra, J.D., Escalona-Segura, G., \& Vargas-Contreras, J.A. (2021). Refugios diurnos del murciélago Rhynchonycteris naso (Chiroptera: Emballonuridae) en Laguna de Términos, Campeche, México. Revista de Biología Tropical, 69(1), 274-290. DOI 10.15517/rbt.v69i1.43595 
La amplia variedad de refugios diurnos utilizados por murciélagos representa un complejo proceso de selección para cubrir requerimientos tanto ecológicos como fisiológicos. Algunos de los factores que afectan dicha selección son la abundancia y disponibilidad de sitios de refugio, flujo interno de aire, temperatura, intensidad de luz, seguridad ante potenciales depredadores y proximidad a zonas de forrajeo (Kunz, 1982; Rodríguez-Durán, 2003; Ávila-Flores \& Medellín, 2004; de Sousa Barros, Bernard, \& Lopes Ferreira, 2020). Por lo anterior, este proceso resulta crucial para los murciélagos debido a que influye en la ecología, organización social y supervivencia de sus poblaciones (Kunz, 1982; Kunz \& Lumsden, 2003).

Los refugios, en la mayoría de las especies de murciélagos, están asociados a lugares ocultos o de difícil acceso, particularmente durante el día, tales como cuevas o cavidades en árboles (Villa-R, 1966; Kunz, 1982; Kunz \& Lumsden, 2003; Breviglieri \& Uieda, 2014), mientras que otras se refugian en lugares expuestos como troncos o ramas en los árboles (Villa-R, 1966; Kunz, 1982; Simmons \& Voss, 1998; Fenton et al., 2001; Knörnschild, Harview, Moseley, \& von Helversen, 2009). Por ejemplo, los murciélagos pertenecientes a la familia Emballonuridae suelen refugiarse en lugares relativamente expuestos e iluminados, como la corteza de árboles, troncos caídos, ramas y debajo de puentes (Bradbury \& Vehrencamp, 1976; Reid, 1997; Fenton et al., 2001). Sin embargo, su postura de percha y los patrones de coloración de su pelaje, les proveen protección ante posibles depredadores (Goodwin \& Greenhall, 1961; Bradbury \& Emmons, 1974; Bradbury \& Vehrencamp, 1976). Dentro de este grupo, la especie que ocupa refugios altamente expuestos a la intemperie es el murciélago narigón Rhynchonycteris naso (Wied-Neuwied, 1820), el cual es físicamente críptico y se refugia sobre los troncos expuestos de árboles y debajo de puentes. La coloración de su pelaje lanudo y moteado con dos líneas pálidas en el dorso y mechones de pelo pálido en los antebrazos, le confieren apariencia de musgo o liquen, lo que le permite pasar desapercibido ante los depredadores, inclusive emulan ser objetos movidos por el viento al llevar a cabo balanceos sincrónicos (Goodwin \& Greenhall, 1961; Plumpton \& Jones, 1992; O'Farrell \& Miller, 1997; Knörnschild et al., 2009).

Rhynchonycteris naso es un pequeño murciélago insectívoro neotropical, catalogado dentro de la Norma Oficial Mexicana-059-SEMARNAT-2010 como sujeta a protección especial (Pr; SEMARNAT, 2010). Se distribuye a lo largo de América Central y a la mitad norte de América del Sur, que incluye el sureste de Brasil, Perú y el norte de Bolivia (Plumpton \& Jones, 1992; Ceballos \& Oliva, 2005; Medellín, Arita, \& Sánchez, 2007). Además de los requerimientos de percha mencionados anteriormente, una peculiaridad de esta especie es que sus refugios se encuentran sobre ríos, arroyos y lagunas (Bradbury \& Emmons, 1974; Plumpton \& Jones, 1992; Emmons \& Feer, 1997).

La dieta de $R$. naso se compone de dípteros (orden Diptera), principalmente quironómidos (familia Chironomidae) y mosquitos (familia Culicidae), y en menor medida de coleópteros (orden Coleoptera) y tricópteros (orden Trichoptera), los cuales captura sobre los cuerpos de agua cercanos a su refugio (Bradbury \& Vehrencamp, 1976; Plumpton \& Jones, 1992; Estrada-Villegas, Meyer, \& Kalko, 2010). Algunos análisis muestran que estos grupos de insectos son los más abundantes en las zonas en las que $R$. naso forrajea, además de que los coleópteros y dípteros presentan menor variación estacional a lo largo del año, lo que les confiere una mayor disponibilidad temporal, aunque sus mayores picos son al final de la temporada de secas y durante la temporada de lluvias (Bradbury \& Vehrencamp, 1976; Frith \& Frith, 1985; Vebrová, Nieuwenhuijzen, Koláŕ, \& Boukal, 2018).

La distribución y abundancia de estos insectos tiene una relación importante con componentes ambientales, por ejemplo, la temperatura y velocidad del viento pueden afectar su dispersión nocturna, mientras que algunos parámetros físicoquímicos del agua, 
tales como el pH y la salinidad, pueden afectar su abundancia en el sitio (Navarrete-Salgado, Fernández-Guillermo, \& Contreras-Rivero, 2004; Waldbauer, 2006; Al-Shami, Rawi, HassanAhmad, \& Nor, 2010; Vebrová et al., 2018). Por lo tanto, si las características fisicoquímicas del agua afectan la disponibilidad de los insectos presa, se podría generar un efecto indirecto sobre la abundancia o la presencia de los murciélagos narigones que dependen de ellos, como ha sido observado en otras especies de murciélagos (Cain, Luoma, Carter, \& Fend, 1992; Racey, 1998; Russ \& Montgomery, 2002; Jones, Jacobs, Kunz, Willig, \& Racey, 2009; Korine, Adams, Shamir, \& Gross, 2015).

Existen diversos estudios que evalúan la relación entre las características bióticas y abióticas de los cuerpos de agua y la actividad de forrajeo de los murciélagos insectívoros (Vaughan, Jones, \& Harris, 1996; Biscardi et al., 2007; Kalcounis-Rueppell, Payne, Huff, \& Boyko, 2007; Razgour, Korine, \& Saltz, 2010; Korine et al., 2015). Algunos de ellos consideran la calidad del agua como elemento determinante en la abundancia de murciélagos y toman como referencia la presencia de macroinvertebrados bioindicadores (Biscardi et al., 2007), o presas potenciales en plantas de tratamiento de aguas residuales (Kalcounis-Rueppell et al., 2007; de Conno et al., 2018). Dentro de estas publicaciones, se menciona que los niveles de algunos parámetros fisicoquímicos del agua, como el pH, varían entre 8 y 9 en los lugares donde forrajean los murciélagos, lo que indica que son ligeramente alcalinos (Korine et al., 2015; Laverty \& Berger, 2020). Sin embargo, estos estudios explican la actividad de forrajeo en cuerpos de agua con diferentes características, sin que, hasta el momento, exista un estudio que evalúe la relación de estos factores con los refugios de murciélagos insectívoros, sobre todo para aquellas especies fuertemente asociadas a cuerpos de agua, como es el caso del murciélago narigón.

Por ello, el propósito de este trabajo fue 1) ubicar y describir los refugios diurnos del murciélago narigón y, 2) evaluar la relación del tamaño del grupo de murciélagos con las características de los refugios y los parámetros fisicoquímicos del agua donde habitan. Hipotetizamos que, el tamaño del grupo por refugio estará relacionado con los parámetros fisicoquímicos del agua que benefician la presencia de sus presas, con lo cual se espera que parámetros como la salinidad y el $\mathrm{pH}$, tengan un efecto en el tamaño del grupo de Rhynchonycteris naso, debido a que la presencia de sus insectos presa podría verse afectada por la variación de estos parámetros (Williams, Boulton, \& Taaffe, 1990; Cain et al., 1992; Al-Shami et al., 2010).

\section{MATERIALES Y MÉTODOS}

Área de estudio: El Área de Protección de Flora y Fauna "Laguna de Términos" (APFFLT), cuenta con una superficie de 706 147 ha y está ubicada en la porción sur de la zona costera del estado de Campeche en los municipios de Carmen, Palizada y Champo-

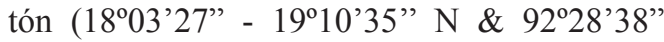
- 9103'16” O) (Fig. 1) (INE-SEMARNAP, 1997). Presenta tres tipos de clima: cálido subhúmedo intermedio con lluvias en verano, cálido subhúmedo con mayor humedad y cálido húmedo con abundantes lluvias en verano. Se definen tres estaciones climáticas: secas (febrero-mayo), lluvias (junio-septiembre) y nortes (octubre-enero) (Yáñez-Arancibia \& Day, 1982; de la Lanza, Ramírez-García, YvesF, \& Alcántara, 1993; INE-SEMARNAP, 1997; García, 2004).

El APFFLT está designado como sitio Ramsar y se encuentra en la región Hidrológica Número 30 Grijalva-Usumacinta, donde se concentra la mayor cantidad de ríos y cuerpos de agua en la entidad (INE-SEMARNAP, 1997; Ramsar, 2020). Además de que es la de mayor importancia en nuestro país, se caracteriza por la interrupción de ríos, arroyos y lagunas, así como zonas de inundación (INE-SEMARNAP, 1997). Mantiene un amplio mosaico de asociaciones vegetales terrestres y acuáticas como la vegetación de dunas costeras y manglar, vegetación de pantano como tular, carrizal y popal, selva baja inundable, matorral espinoso inundable, matorral inerme inundable, 

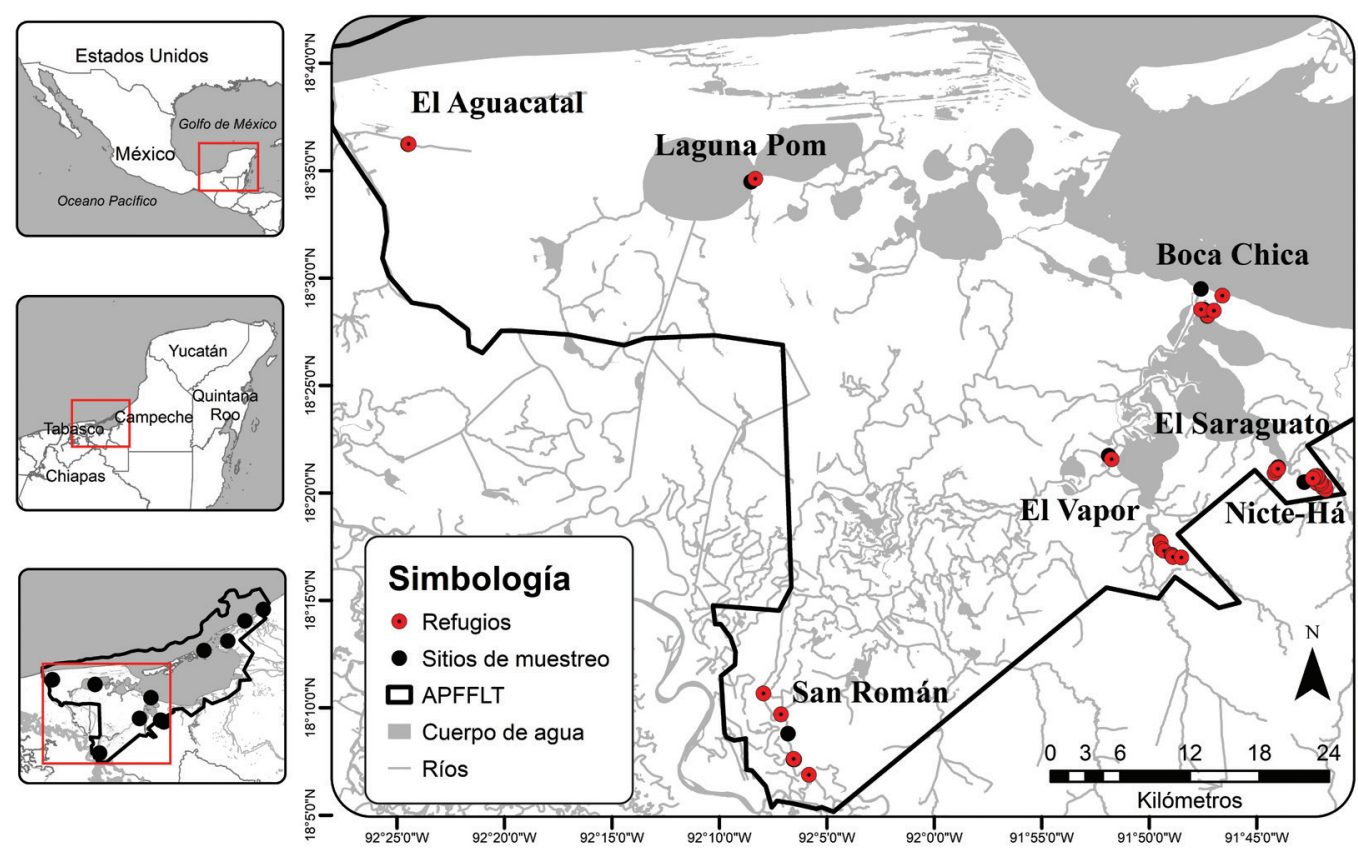

Fig. 1. Distribución de los refugios diurnos del murciélago narigón, Rhynchonycteris naso (ć́rculos rojos) en diferentes sitios de muestreo (círculos negros) dentro del Área de Protección de Flora y Fauna Laguna de Términos (APFFLT) en Campeche, México.

Fig. 1. Distribution of the diurnal roost of the Proboscis bat (red circles) in different sampling sites (black circles) within the Laguna de Términos Flora and Fauna Protection Area (APFFLT) in Campeche, Mexico.

vegetación ribereña, selva alta, selva mediana, vegetación secundaria y pastos marinos (INESEMARNAP, 1997). El APFFLT cuenta con una extensión de manglar mayor a $700 \mathrm{~km}^{2}$, lo que representa el $61.8 \%$ de la cobertura del manglar del país (de la Lanza et al., 1993; Agraz et al., 2015). La región se divide en cuatro subcuencas: Laguna de Pom y Atasta, Laguna del Este, Río Palizada y, San Pedro y San Pablo (INEGI, 2010). Las tres primeras subcuencas desembocan en el APFFLT, mientras que San Pedro y San Pablo lo hacen en el Golfo de México.

Búsqueda de refugios: De febrero a octubre 2015 se realizaron búsquedas intensivas de refugios diurnos del murciélago narigón y se recorrió una distancia aproximada de 186.58 $\mathrm{km}$ en once sitios correspondientes a ríos, arroyos, lagos y lagunas ubicadas dentro del APFFLT: San Francisco, Estero de Sabancuy,
Nohan, Laguna Pom, El Aguacatal, El Vapor, Rancho R1, San Román, Nicte-Há, El Saraguato y Boca Chica (Fig. 1). En cada sitio, se llevó a cabo un recorrido en lancha para realizar la búsqueda y localización de refugios diurnos en ambos lados del cuerpo de agua. Los recorridos fueron durante las horas más frescas del día, principalmente una o dos horas después del amanecer $y$, en ocasiones, cuatro horas antes de la puesta del sol. Los ríos y arroyos se recorrieron a una distancia mínima de $15 \mathrm{~m}$ de la orilla para evitar perturbar a los murciélagos y minimizar los posibles efectos de la presencia de los observadores, cuando el arroyo era muy estrecho, el recorrido se hizo con motor apagado y se transitó por el medio del afluente. Adicional a la observación directa y debido a que, en ocasiones, los individuos no eran detectables a simple vista, se usaron binoculares y cámaras fotográficas. 
Una vez detectado un refugio, se apagó el motor de la lancha y se procedió a acercarse al refugio a una distancia mínima de $5 \mathrm{~m}$ evitando perturbar a los murciélagos. En ese punto, se tomaron fotografías y se llevó a cabo la caracterización de los refugios. Además, se realizó el conteo de individuos en el refugio, los bosquejos del acomodo de los individuos en el refugio y la medición de los parámetros fisicoquímicos del agua, como se describe en los párrafos siguientes. La posición de cada refugio fue registrada con un geoposicionador satelital marca Garmin (eTrex10) bajo un sistema de coordenadas Transversal de Mercator (UTM) proyectadas en el datum WGS84 (zona 15Q) a una precisión de $5 \mathrm{~m}$. La distancia entre cada refugio al refugio más cercano se calculó utilizando la herramienta "Point distance" de ArcMap (v.10.4.1, ESRI).

Caracterización de los refugios: Los refugios fueron catalogados como naturales cuando se encontraron sobre árboles o restos de estos, como troncos, y artificiales cuando fueron observados en estructuras de origen antrópico. En el caso de los refugios naturales, se determinó la especie vegetal en la cual se encontraron perchados los murciélagos, mientras que para los refugios artificiales se determinó el material de la estructura de percha. A partir de las fotografías tomadas para cada refugio, se calculó el diámetro de las especies vegetales dónde se encontraban los murciélagos, utilizando la longitud del antebrazo del murciélago narigón $(40 \mathrm{~mm})$ como escala de referencia (Plumpton \& Jones, 1992). Las mediciones para calcular el diámetro se realizaron con el programa de procesamiento de imagen ImageJ (National Institutes of Health, Bethesda MD, USA). El tipo de vegetación en los alrededores del refugio se asignó siguiendo las clasificaciones de la Capa de Uso del Suelo y Vegetación escala 1:250000 Serie VI de INEGI (INEGI, 2014) y se verificó en campo. Se evaluó visualmente la presencia o ausencia de cobertura a partir de la existencia de dosel y estructuras sobre los refugios localizados.
Tamaño del grupo y acomodo de murciélagos en el refugio: El tamaño del grupo se definió como el valor máximo de murciélagos observados por refugio al momento del encuentro. Para definir la forma del acomodo de los individuos en el refugio definimos tres categorías diferentes: 1) al azar cuando la posición de los individuos no seguía un patrón visible; 2) uniforme, cuando la formación de los individuos seguía un patrón lineal; y 3) agregada cuando los individuos formaban varios subgrupos en el mismo refugio (Andriolo, Rodrigues, Zerbini, \& Barrio, 2010).

\section{Medición de parámetros fisicoquími-} cos. Por cada refugio localizado se realizaron mediciones in situ con una sonda multiparamétrica HANNA (modelo HI929829), previamente calibrada en el laboratorio. Se sumergió la sonda a una profundidad de $1 \mathrm{~m}$ para tener el registro de las variables salinidad (ups) y potencial de Hidrógeno $(\mathrm{pH})$. En los sitios recorridos, donde no se encontraron refugios, se realizaron de tres a cuatro mediciones aleatorias de control a lo largo del recorrido, dependiendo de la longitud del río. De acuerdo con sus niveles de salinidad y siguiendo la propuesta de Venice (Anon, 1958), el agua se clasificó en dulce $(<0.5$ ups), oligohalina (0.5-5 ups), mesohalina (5-18 ups), polihalina (18-30 ups), euhalina ( $>30$ ups) e hiperhalina ( $>40$ ups).

Análisis de resultados: Para evaluar el nivel de agrupamiento de los refugios en cada uno de los sitios, se llevó a cabo un análisis de agrupamiento jerárquico siguiendo el método de Ward (Kauffman \& Rousseuw, 1990), para el cual se utilizaron las distancias más cercanas entre cada refugio. El método arroja un coeficiente de aglomeramiento entre cero y uno, donde los valores cercanos a uno indican agrupamiento.

Se ajustaron Modelos Lineales Generalizados (GLMs) bajo una distribución del error del tipo binomial negativa (Crawley, 2013), para investigar los factores que influencian el tamaño del grupo en un determinado refugio. Las variables que se consideraron fueron: el tipo 
de refugio, el tipo de vegetación, la cobertura, la distancia entre refugios, la salinidad y el $\mathrm{pH}$. La variable diámetro de las especies vegetales se evaluó por separado debido a que incluyó únicamente a los refugios naturales. El modelo mínimo adecuado fue el que obtuvo el menor valor de criterio de información de Akaike (AIC) en una selección paso a paso (stepwise) (Crawley, 2013).

Todos los análisis estadísticos emplearon un valor de Alpha de 0.05 y se llevaron a cabo utilizando el programa $\mathrm{R}$ versión 1.1.456 ( $\mathrm{R}$ Core Team, 2017) y las paqueterías cluster (Maechler, Rousseeuw, Struyf, Hubert, \& Hornik, 2019), ggplot2 (Wickham, 2016) y MASS (Venables \& Ripley, 2002). Los modelos fueron validados a partir de la examinación de los residuales.

\section{RESULTADOS}

Búsqueda de los refugios: En total encontramos 48 refugios diurnos dentro de la cuenca Grijalva-Usumacinta, en las subcuencas Laguna Pom, San Pedro y San Pablo, Río Palizada y Río del Este.

Caracterización de los refugios: De los 48 refugios, 43 se ubicaron en sustratos naturales $(89.58 \%)$ y cinco en artificiales (10.42 $\%)$. Todos fueron localizados sobre cuerpos de agua (Fig. 1). Los refugios naturales los encontramos sobre troncos $\operatorname{secos}(\mathrm{N}=3)$ y la corteza de árboles de cinco especies vegetales: mangle rojo (Rhizophora mangle; $\mathrm{N}=17$ ), zapote de agua (Pachira aquatica; $\mathrm{N}=12$ ), mangle blanco (Laguncularia racemosa; $\mathrm{N}=$ 8), mangle botoncillo (Conocarpus erectus; $\mathrm{N}$ $=2$ ) y mangle negro (Avicennia germinans; $\mathrm{N}$ $=1)$. Los refugios artificiales los ubicamos en un puente de metal $(\mathrm{N}=1)$ y en los pilares de un puente de concreto $(\mathrm{N}=4)$.

En cuanto al tipo de vegetación circundante, encontramos un mayor número de refugios en el manglar (37.5\%), seguido por la selva mediana subperennifolia con vegetación secundaria arbórea (SMSP_VSA, $27.2 \%$ ), pastizal $(18.75 \%)$ y vegetación secundaria arbórea
(VSA, $16.66 \%$ ) (Tabla 1). El $79.17 \%$ de los refugios localizados presentaron algún tipo de cobertura (natural o artificial) que les ofrecía protección contra los rayos directos del sol.

Los refugios diurnos del murciélago narigón se encontraron agrupados (coeficiente de aglomeración $>0.81$ ) en cinco de los 11 sitios a una distancia inter-refugio promedio ( \pm error estándar) de $619.83 \pm 226.49 \mathrm{~m}$, siendo los sitios El Vapor y San Román los que presentaron refugios más distantes (1 257.08 \pm 812.99 y $1027.08 \pm 812.99 \mathrm{~m}$, respectivamente), mientras que en los sitios Nicte-Há y El Saraguato los refugios se encontraron más cercanos unos de otros $(221.25 \pm 28.53$ y $89.12 \pm 33.84 \mathrm{~m})$ (Tabla 1).

Tamaño del grupo y acomodo de murciélagos en el refugio: El número total de individuos contabilizados en los refugios fue de 341 , de los cuales 228 estuvieron en refugios naturales y 113 en refugios artificiales. El promedio general de individuos por refugio fue de $5.30 \pm 0.48$ individuos para los refugios naturales, mientras que en los refugios artificiales fue de $22.6 \pm 9.54$ individuos. Sólo tres hembras fueron observadas con una cría en el mes de marzo en el refugio Laguna Pom. En cuanto al acomodo de los murciélagos en los refugios, en los naturales fue principalmente uniforme (39 refugios) y al azar (cuatro), en tanto que, en los cinco refugios artificiales, la disposición de los individuos fue agregada. Los refugios con mayor tamaño del grupo promedio presentaron un acomodo agregado, mientras que, al disminuir el tamaño del grupo, el acomodo tendió a ser uniforme y al azar (Fig. 2).

Tamaño del grupo y su relación con las características del refugio: El tamaño del grupo por refugio estuvo explicado por el tipo de refugio $\left(\chi_{(0.05,46)}^{2}=39.64, \mathrm{P}<0.001\right)$, el tipo de vegetación en sus alrededores $\left(\chi^{2}{ }_{(0.05}\right.$, $\left.{ }_{43)}=10.04, \mathrm{P}=0.018\right) \mathrm{y}$ el diámetro del tronco de las especies vegetales $\left(\chi_{(0.05,41)}^{2}=3.99, \mathrm{P}\right.$ $=0.046)$. En los refugios artificiales, el tamaño del grupo fue significativamente mayor en comparación con los refugios naturales 
TABLA 1

Resumen de las variables asociadas a los refugios diurnos de Rhynchonycteris naso

TABLE 1

Summary of variables associated with diurnal roost of Rhynchonycteris naso

\begin{tabular}{lcccccc}
$\begin{array}{c}\text { Sitio de } \\
\text { muestreo }\end{array}$ & $\begin{array}{c}\text { Número de } \\
\text { refugios }\end{array}$ & $\begin{array}{c}\text { Número total de } \\
\text { individuos }\end{array}$ & $\begin{array}{c}\text { Tamaño del } \\
\text { grupo }(\overline{\mathrm{X}} \pm \mathrm{EE})\end{array}$ & $\begin{array}{c}\text { Tipo de } \\
\text { vegetación }\end{array}$ & $\begin{array}{c}\text { Distancia entre } \\
\text { refugios }(\mathrm{m}) \\
(\overline{\mathrm{X}} \pm \mathrm{EE})\end{array}$ & $\begin{array}{c}\text { Coeficiente de } \\
\text { aglomeración }\end{array}$ \\
Laguna Pom & 1 & 10 & 10 & Manglar & - & - \\
El Aguacatal & 1 & 40 & 40 & Manglar & - & - \\
El Vapor & 9 & 63 & $7 \pm 1.55$ & Manglar & $1257.08 \pm 812.99$ & 0.87 \\
San Román & 8 & 95 & $11.87 \pm 5.62$ & Pastizal & $1027.31 \pm 494.09$ & 0.89 \\
Nicte-Há & 13 & 72 & $5.53 \pm 0.63$ & SMSP_VSA & $221.25 \pm 28.53$ & 0.92 \\
El Saraguato & 8 & 21 & $2.62 \pm 0.37$ & VSA & $89.12 \pm 33.84$ & 0.91 \\
Boca Chica & 8 & 40 & $5 \pm 1.01$ & Manglar & $504.38 \pm 177.21$ & 0.82 \\
\hline
\end{tabular}

Promedio $(\overline{\mathrm{X}})$, error estándar $(\mathrm{EE})$. Tipo de vegetación: selva mediana subperennifolia con vegetación secundaria arbórea (SMSP_VSA), vegetación secundaria arbórea (VSA).

Average $(\overline{\mathrm{X}})$, standard error (EE). Vegetation type: medium semi-humid forest with secondary tree vegetation (SMSP VSA), secondary tree vegetation (VSA).

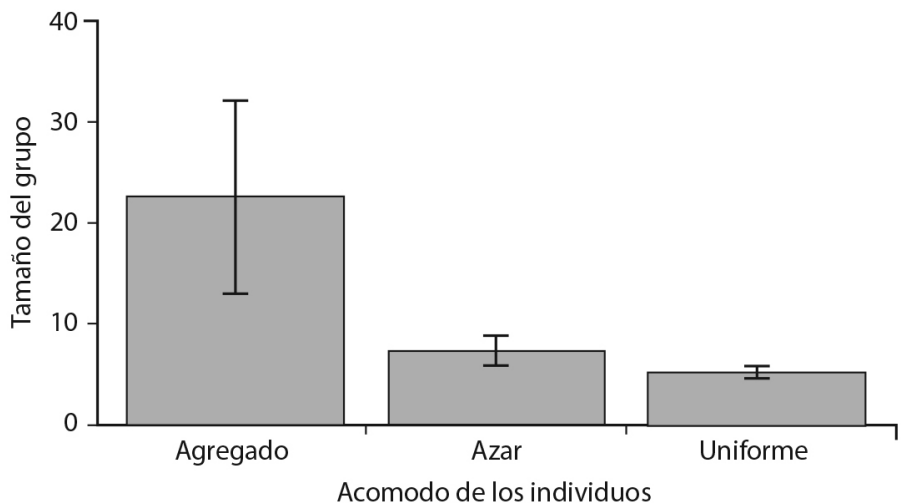

Fig. 2. Tamaño del grupo promedio según el acomodo de los individuos en el refugio. Las líneas verticales en cada columna muestran el error estándar alrededor de la media.

Fig. 2. Average group size based on the distribution of individuals in the roost. The vertical lines in each column show a standard error around the mean.

(Tabla 2). La vegetación de manglar albergó los refugios con el mayor tamaño del grupo, mientras que la VSA fue el tipo de vegetación donde encontramos refugios con el menor número de individuos (Tabla 2). Por otro lado, el diámetro del tronco de las especies vegetales (únicamente para el caso de los refugios naturales), tuvo un efecto positivo y significativo en el tamaño del grupo por refugio (Tabla 2) (Fig. 3).
Salinidad y pH: En promedio, la salinidad registrada en los refugios diurnos fue de $0.40 \pm 0.07$ ups $(\mathrm{N}=48)$. El $83.3 \%$ de los refugios se localizaron sobre cuerpos de agua dulce, mientras que el $16.6 \%$ en cuerpos de agua con características oligohalinas. No se encontró asociación del tamaño del grupo por refugio con la salinidad. En promedio, la salinidad registrada en los sitios recorridos donde no se observaron refugios diurnos fue de 26.18 
TABLA 2

Parámetros de regresión estimados (Est), error estándar (EE), estadístico de prueba $(z)$ y probabilidad (P), para cada uno de los modelos ajustados (M1 y M2)

TABLE 2

Estimated regression parameters (Est), standard error (EE), the test statistic (z), and probability (P), for each of the adjusted models (M1 and M2)

\begin{tabular}{|c|c|c|c|c|c|}
\hline $\mathrm{E}$ & Variables & Est & $\mathrm{EE}$ & $z$ & $\mathrm{P}$ \\
\hline \multirow[t]{6}{*}{$\mathrm{Bn}$} & M1. Tamaño del grupo $\sim$ Tipo de refugio + Tipo de vegetación & & & & \\
\hline & Intercepto & 3.3964 & 0.3285 & 10.339 & $<0.001$ \\
\hline & Tipo de refugio (natural) & -1.4999 & 0.3206 & -4.679 & $<0.001$ \\
\hline & Tipo de vegetación (pastizal) & -0.3977 & 0.2905 & -1.369 & 0.17093 \\
\hline & Tipo de vegetación (SMSP_VSA) & -0.1848 & 0.2298 & -0.804 & 0.42118 \\
\hline & Tipo de vegetación (VSA) & -0.9314 & 0.3124 & -2.982 & $<0.01$ \\
\hline \multirow[t]{3}{*}{$\mathrm{Bn}$} & M2. Tamaño del grupo $\sim$ Diámetro de las especies vegetales & & & & \\
\hline & Intercepto & 1.4164 & 0.1502 & 9.432 & $<0.001$ \\
\hline & Diámetro de las especies vegetales & 0.0084 & 0.0041 & 2.043 & $<0.05$ \\
\hline
\end{tabular}

Distribución del error (E): Bn (binomial negativo). Tipo de vegetación: selva mediana subperennifolia con vegetación secundaria arbórea (SMSP_VSA), vegetación secundaria arbórea (VSA).

Error distribution (E): Bn (negative binomial). Vegetation type: medium semi-humid forest with secondary tree vegetation (SMSP_VSA), secondary tree vegetation (VSA).

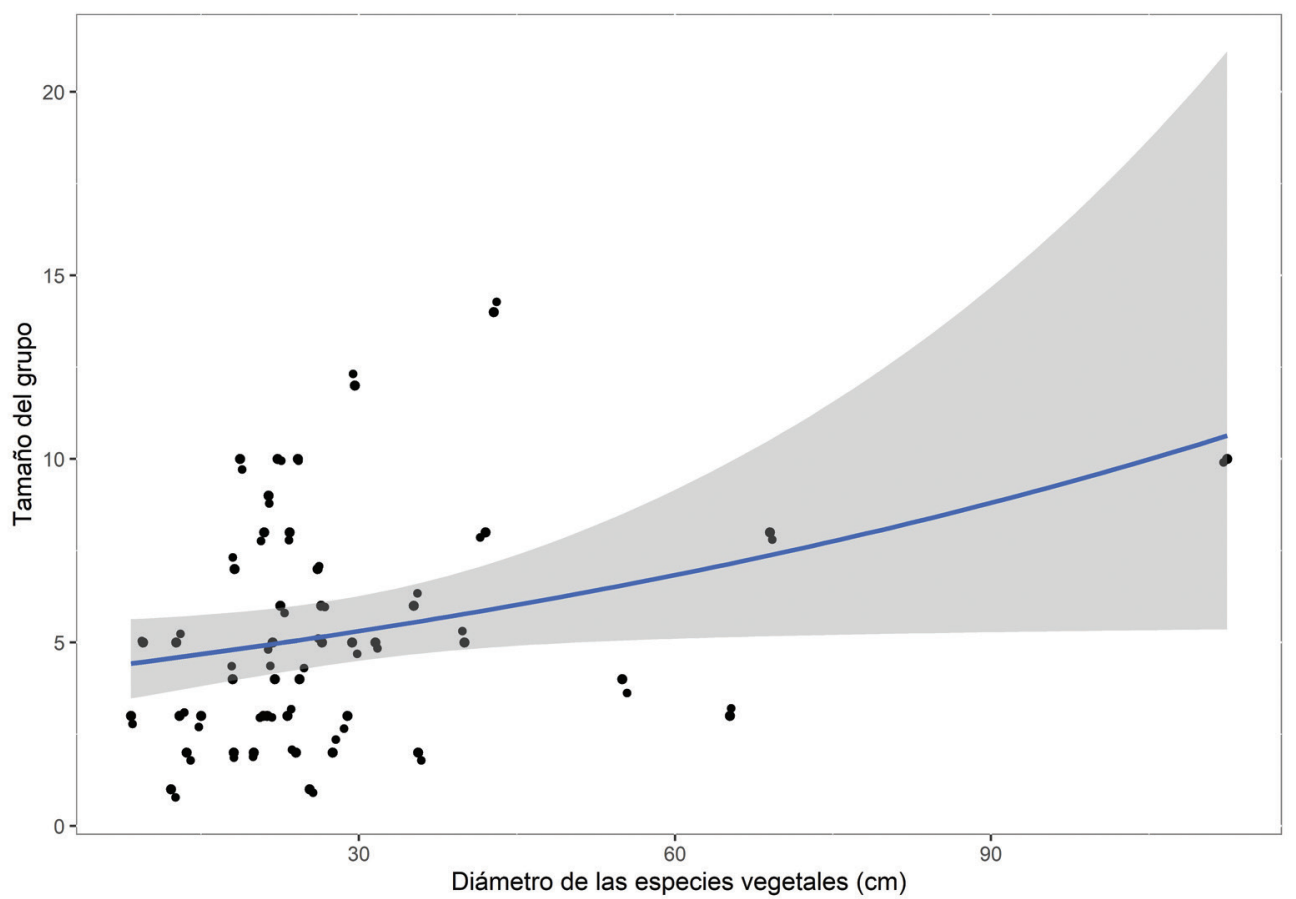

Fig. 3. Efecto del diámetro del tronco de las especies vegetales con el tamaño del grupo por refugio. El área sombreada representa los intervalos de confianza al $95 \%$.

Fig. 3. Effect of the trunk diameter of plant species on the group size per roost. The shaded area represents the $95 \%$ confidence intervals. 
\pm 4.12 ups $(\mathrm{N}=14)$. El pH registrado en los sitios con refugios diurnos fue de $6.19 \pm 0.15$ $(\mathrm{N}=48)$, lo que indica que los cuerpos de agua son ligeramente ácidos. En los sitios recorridos donde no se observaron refugios fue de $7.40 \pm$ $0.19(\mathrm{~N}=14)$, lo que indica que los cuerpos de agua son neutros. No se encontró asociación del tamaño del grupo por refugio con el $\mathrm{pH}$.

\section{DISCUSIÓN}

El presente estudio es el primero en describir los refugios diurnos de $R$. naso e investigar la relación del tamaño del grupo con las características de sus refugios y los parámetros fisicoquímicos del agua. Encontramos a $R$. naso principalmente en sustratos naturales rodeados por vegetación de manglar asociados a bordes de ríos, arroyos y lagunas. El tamaño del grupo por refugio se asoció principalmente al tipo del refugio, al tipo de vegetación y al diámetro del tronco de las especies vegetales. Aunque estadísticamente no se encontró una relación entre los parámetros físicoquímicos del agua y el tamaño del grupo, en términos de porcentaje, más del $80 \%$ de los refugios se encontraron sobre cuerpos de agua con bajos niveles de salinidad ( $<0.5$ ups) y la mayoría de ellos ligeramente ácidos $(\mathrm{pH}<7)$, que constituyen condiciones adecuadas para el establecimiento de comunidades de insectos (Navarrete-Salgado et al., 2004; Waldbauer, 2006; Al-Shami et al., 2010; Vebrová et al., 2018), lo que podría favorecer el forrajeo óptimo de los individuos (Stephens \& Krebs, 1986; Nogueira \& Pol, 1998).

Caracterización de refugios: El APFFLT se caracteriza por la presencia de grandes extensiones de manglar (de la Lanza et al., 1993; INEGI, 2014; Agraz et al., 2015), lo que se traduce en una alta dominancia de especies de mangle y, por lo tanto, fue una especie recurrentemente usada como sitio de refugio por el murciélago narigón y correspondió al tipo de vegetación que albergó los refugios con el mayor tamaño de grupo. Por lo anterior, la vegetación de manglar es un hábitat importante para el establecimiento de esta especie en el APFFLT, puesto que también le confiere una mayor protección contra depredadores debido a las intrincadas raíces que presentan, además de que ofrecen abundancia de presas potenciales (Fernandes, 2000; Vannucci, 2001; Hogarth, 2007; Salas, 2010; McConville, Law, \& Mahony, 2013; Soares et al., 2016). Esto resulta crucial en términos de conservación de la especie, ya que la región del APFFLT representa el límite norteño de su distribución potencial (Ceballos, Blanco, González, \& Martínez, 2006), por lo que la pérdida de cobertura de manglar podría tener efectos negativos en sus poblaciones, provocando una disminución en su rango de distribución, como ha sucedido con otros grupos zoológicos (Pacifici et al., 2020).

Sin embargo, aun cuando existe vegetación de manglar en el noreste del área de estudio, no se localizaron refugios diurnos de la especie. Esta puede ser una razón más para suponer que los murciélagos requieren de hábitats ribereños y cuerpos de agua dulce, los cuales son escasos en la porción norte del APFFLT; esto es apoyado por los registros de mayor salinidad en esa misma porción (Gold-Bouchot, NoreñaBarroso, \& Zapata-Pérez, 1995).

Los refugios de $R$. naso se reportan sobre especies de árboles característicos de selvas bajas o selvas medianas perturbadas y especies asociadas a hábitats inundados, como es el caso de Albizia, Pterocarpus, Salix y Haematoxylum (Fabaceae; Bradbury \& Emmons, 1974; Salas, Viteri, Zambrano, Benavides, \& Carvajal, 2013; Uc-Cua, 2017). En el estado de Tabasco, dentro de la Reserva de la Biosfera Pantanos de Centla, se ha reportado el uso de Salix humboldtiana y Bucida buceras con mayor frecuencia, en comparación con el manglar (Uc-Cua, 2017), esto puede deberse a que el manglar constituye solo el $2 \%$ de la superficie en dicha reserva, ya que sus límites no consideran zona costera, mientras que en el APFFLT, esta vegetación representa más del $10 \%$ de la superficie del área protegida (INESEMARNAP, 1997; SEMARNAP, 2000).

Los refugios que estuvieron dentro de zonas ribereñas con vegetación secundaria arbórea, selva mediana subperennifolia y 
pastizales, se encontraron en Pachira aquatica, la cual, además de ser similar fisonómicamente a las especies de mangle (por presentar en el tallo contrafuertes y raíces adventicias), es considerada como un indicador del límite de distribución de la vegetación de manglar (Infante-Mata, Moreno-Casasola, \& MaderoVega, 2014). Por lo tanto, es posible que $R$. naso presente cierto grado de plasticidad en la selección de sus refugios diurnos y esta selección esté más regulada por su disponibilidad y por algún rasgo propio del tronco o árbol, por ejemplo, especies arbóreas con capacidades para crecer en zonas inundadas.

Cabe mencionar que algunos de los refugios diurnos se observaron en sitios con impacto humano constante (p. ej. tráfico diario de lanchas o puentes con tráfico de vehículos), lo que podría sugerir que la perturbación antrópica directa es un elemento al que pueden acostumbrarse, como ha sido reportado para una colonia localizada en la cornisa de una cabina de madera utilizada como estación de monitoreo de fauna, donde existe una actividad humana constante y donde se conoce que los murciélagos lo han ocupado al menos por 15 años (Günther et al., 2016). Sin embargo, la fragmentación y destrucción del hábitat son procesos que podrían poner en mayor riesgo la presencia de la especie, principalmente por actividades agrícolas en las que se emplean diferentes componentes químicos como herbicidas, pesticidas y fertilizantes. Por ejemplo, en la zona noreste del APFFLT se ha encontrado un significativo descenso de la actividad de colinesterasas en ratones, asociadas al uso de pesticidas organofosforados (Chi-Coyoc et al., 2016). En este sentido, los pesticidas han demostrado tener efectos negativos en la actividad y presencia de murciélagos, particularmente insectívoros (Stahlschmidt \& Brühl, 2012; Lilley et al., 2013; Korine et al., 2015), así como en la biomasa y diversidad de insectos (Whitehurst \& Lindsey, 1990; Vaughan, Jones, \& Harris, 1996). Por lo anterior, resulta relevante un estudio enfocado a la exposición a contaminantes y la calidad del agua en esta especie, debido a su asociación a cuerpos de agua.

Para poder ocupar refugios expuestos al medio, los murciélagos, como muchas especies de mamíferos, presentan patrones de coloración asociados a un comportamiento críptico, ya sea para evitar la depredación o para sorprender a la presa (Merilaita, Tuomi, \& Jormalainen, 1999; Caro, 2005). Evolutivamente, se ha documentado que la presencia de patrones de pigmentación en el pelaje de algunos murciélagos, tales como líneas o manchas claras contrastantes con su pelaje de fondo, está asociado a los hábitos de refugio de los individuos y al tamaño de sus colonias (Santana, Dial, Eiting, \& Alfaro, 2011). En este contexto, las líneas claras dorsales y los mechones de pelo en los antebrazos del murciélago narigón le confieren un mecanismo de protección contra depredadores al refugiarse en sitios expuestos de manera parcial, como en la mayoría de los registros en el presente estudio. Sin embargo, también se han encontrado grupos de individuos utilizando árboles totalmente expuestos, como lo reportado en este estudio y por otros autores (Dalquest, 1957; Bradbury \& Emmons, 1974; Knörnschild et al., 2009), aunque de manera menos frecuente. Lo anterior puede tener repercusiones tanto en su tasa de depredación, como fisiológicas en la regulación de su temperatura, aspecto que ha sido poco explorado para esta especie (p. ej. Wilde, Günther, Mayer, Knörnschild, \& Nagy, 2018).

La presencia de $R$. naso en refugios artificiales a pesar de la disponibilidad de refugios naturales en los alrededores, podría deberse a que estos, además de que cumplen con las condiciones necesarias para servir como refugios, son más propicios que los naturales debido a que ofrecen una mayor estabilidad y protección a la colonia, incluso pueden soportar un mayor número de individuos (Davis \& Cockrum, 1963; Nogueira \& Pol, 1998; Chambers, Alm, Siders, \& Rabe, 2002; Mering \& Chambers, 2014), lo cual constatamos en nuestro estudio en donde encontramos las colonias más grandes de murciélagos en dichos refugios. 
En los refugios donde se ha observado a $R$. naso se ha documentado que forman pequeños grupos de individuos alineados verticalmente (Dalquest, 1957; Goodwin \& Greenhall, 1961; Knörnschild et al., 2009), acomodo que en este estudio se denominó patrón uniforme. Observamos ese mismo patrón en refugios con un máximo de 14 individuos. En grupos más numerosos ( $>10$ individuos) se ha observado un patrón oval en la disposición espacial de los individuos (Nogueira \& Pol, 1998), lo que en este estudio se consideró patrón al azar. Sin embargo, contrario a esto, nosotros observamos este patrón en colonias de tres a diez individuos. En colonias numerosas, se ha observado un arreglo espacial oval de los individuos y la formación de pequeños subgrupos (Dalquest, 1957; Nogueira \& Pol, 1998). En nuestro estudio, observamos la agregación de los individuos formando subgrupos, lo cual coincide con lo observado previamente.

Aunque el tamaño del grupo ha sido un buen predictor del acomodo de los individuos (Dalquest, 1957; Goodwin \& Greenhall, 1961), Knörnschild et al. (2009) observaron que, independientemente del tamaño de la colonia, los murciélagos que percharon en estructuras con un diámetro pequeño como troncos delgados y ramas formaban una línea vertical. Sin embargo, en el presente estudio se observó que, aun cuando los refugios artificiales presentaban un alto número de individuos, estos se mantuvieron siempre agrupados de manera agregada, conformando subgrupos bien diferenciados, por lo que suponemos que el acomodo de los individuos puede obedecer más a una organización social. Este comportamiento ha sido documentado en refugios artificiales, en donde cada subgrupo presenta una alta fidelidad a su sitio de refugio al menos durante el día, mientras que, durante su actividad nocturna, los individuos pueden ocupar otros subgrupos dentro del refugio artificial, siendo las hembras las que más se mueven entre estos (Günther et al., 2016).

Dadas estas interacciones sociales entre grupos, resulta congruente pensar que los refugios se mantienen concentrados en zonas específicas, explicadas por su corta distancia entre refugios vecinos, probablemente formando colonias más grandes, pero subagrupadas, un tipo de organización social conocido como fisión-fusión (Kerth \& König, 1999). Este tipo de organización social podría presentarse en esta especie dentro del APFFLT, en donde encontramos altos coeficientes de agrupamiento. Lo anterior indica que los individuos forman una unidad social conformada por subgrupos de tamaño variable entre los cuales existen interacciones sociales. Este comportamiento ha sido observado en murciélagos que se refugian en cavidades de árboles como el murciélago mayor café (Eptesicus fuscus) en América y el murciélago de Bechstein (Myotis bechsteinii) en Europa (Kerth \& König, 2001; Willis \& Brigham, 2004). Esto también explica que en las estructuras artificiales donde existe una mayor área para ocupar, los murciélagos no formen una colonia grande con individuos entremezclados, sino que mantengan la estructura social subagrupada. Por lo anterior, es importante llevar a cabo estudios sobre la fidelidad de los sitios de refugio y su organización social en esta especie amenazada por las actividades humanas.

Salinidad y pH: Dentro del APFFLT se encuentran sistemas fluvio-lagunares y cuerpos lagunares, que se caracterizan por presentar diferentes niveles de salinidad (Gold-Bouchot et al., 1995; INE-SEMARNAP, 1997), lo cual se pudo notar en los sitios visitados durante este estudio, que tuvieron fluctuaciones de 0.09 a 42.64 ups. El sistema fluvio-lagunar Palizada-del Este se caracterizó por tener los valores de salinidad más bajos, debido a que está constituido por la porción terminal del Río Palizada y las lagunas del Vapor, del Este y San Francisco, que en su conjunto aportan un $70 \%$ del total de agua dulce que ingresa a la Laguna de Términos (Amezcua-Linares \& YáñezArancibia, 1980; INE-SEMARNAP, 1997), consecuentemente, se ubicó el mayor número de refugios e individuos en estos sistemas.

Nuestros resultados muestran que además de su asociación a los cuerpos de agua 
(Dalquest, 1957; Bradbury \& Vehrencamp, 1976; Plumpton \& Jones, 1992; Ceballos \& Oliva, 2005), la presencia de refugios diurnos del murciélago narigón estuvo relacionada con bajas concentraciones de salinidad y poca acidez, lo que podría estar relacionado con la disminución en el costo energético para llegar a estas zonas y con la disponibilidad de alimento (Jackson \& Fisher, 1986; Stephens \& Krebs, 1986). Aun cuando en los bordes de la Laguna de Términos se contaba con vegetación adecuada para la presencia de sus refugios diurnos, no se observó ningún refugio durante los recorridos. Lo anterior podría deberse a los altos niveles de salinidad en esa zona (> 13 ups), ya que el refugio más cercano a este cuerpo de agua se localizó a $404 \mathrm{~m}$ de distancia, donde los niveles de salinidad eran menores a 1.85 ups, lo que podría sugerir que la salinidad es un factor limitante en su presencia y distribución. Por ello, sería necesario ampliar los esfuerzos en el estudio de la relación de la salinidad con la presencia y distribución de sus refugios diurnos, principalmente en zonas costeras, puesto que algunos de sus registros han sido inclusive, en esteros muy cercanos a costa (Salas et al., 2013).

La distribución de los sitios de percha del murciélago narigón y la salinidad del agua, podrían resultar interesantes desde la perspectiva del cambio climático. Algunos modelos predicen una tendencia ascendente en la salinidad en zonas costeras debido al aumento de la temperatura global, lo que significaría una menor disponibilidad de agua dulce (Durack, Wijffels, $\&$ Boyer, 2013). Por lo tanto, una manera de comprender los efectos indirectos asociados al cambio climático es estudiar a aquellas especies cuyos patrones ecológicos y geográficos están asociados a tales variaciones, de manera que puedan ser estudiados y utilizados como especies indicadoras de cambios ambientales. Por ello, estudiar la selección de refugios a largo plazo para una especie con alta dependencia a variables microclimáticas como el murciélago narigón, es una alternativa viable para determinar los efectos del cambio climático.
Finalmente, en términos de conservación, la Unión Internacional para la Conservación de la Naturaleza (IUCN por sus siglas en inglés), reconoce que la tendencia poblacional de $R$. naso es desconocida y que su principal amenaza está relacionada con los cuerpos de agua en donde se refugia y alimenta (Lim \& Miller, 2016). Sin embargo, la coloca como especie de preocupación menor debido a que está ampliamente distribuida (Least Concern). En México, la especie está bajo la categoría Protección Especial (Pr), lo que significa que es una especie con altas necesidades de conservación debido al elevado nivel de amenaza asociado, principalmente, a la pérdida del hábitat y por tanto de sus refugios diurnos (SEMARNAT, 2010). Por lo anterior, la información ofrecida en este documento, representa un importante aporte al conocimiento de la especie y demuestra su alta dependencia a los cuerpos de agua dulce y oligohalina, demostrando ser un buen objeto de estudio en humedales y, principalmente, en sistemas ribereños cuya cobertura forestal está siendo desplazada por actividades antrópicas como la ganadería y la agricultura, que además de la destrucción del hábitat, provocan alteraciones fisicoquímicas en los sistemas fluvio-lagunares donde se presentan.

Declaración de ética: los autores declaran que todos están de acuerdo con esta publicación y que han hecho aportes que justifican su autoría; que no hay conflicto de interés de ningún tipo; y que han cumplido con todos los requisitos y procedimientos éticos y legales pertinentes. Todas las fuentes de financiamiento se detallan plena y claramente en la sección de agradecimientos. El respectivo documento legal firmado se encuentra en los archivos de la revista.

\section{AGRADECIMIENTOS}

Agradecemos a la CONABIO por el financiamiento del proyecto "Inventario de aves y mamíferos en humedales de Laguna de Términos y Pantanos de Centla en Tabasco y Campeche (LH009)", al director de la 
reserva APFFLT, a José Hernández Nava y a los guardaparques del área, a las personas de las comunidades y a los integrantes de este proyecto (tesistas, técnicos e investigadores) por su apoyo en campo, así como a ECOSUR y la Facultad de Ciencias Químico Biológicas de la Universidad Autónoma de Campeche por el apoyo logístico-académico para la realización de la tesis. A los revisores del manuscrito por sus valiosas aportaciones, las cuales enriquecieron el presente documento.

\section{RESUMEN}

Introducción: La selección de refugios diurnos es un proceso crucial para los murciélagos debido a su influencia en la ecología, organización social y supervivencia de sus poblaciones. El murciélago narigón (Rhynchonycteris naso) es una especie protegida por leyes de México, cuyos refugios y zonas de forrajeo, están estrictamente asociados a cuerpos de agua, principalmente ríos. Por lo tanto, cambios en la calidad del agua suponen un riesgo para sus poblaciones. Sin embargo, el conocimiento sobre el estado actual de sus poblaciones y refugios diurnos en México, es escaso. Objetivo: Ubicar y describir los refugios diurnos de $R$. naso y evaluar la relación del tamaño del grupo de murciélagos con las características de los refugios, la salinidad y el pH del agua donde habitan. Métodos: De marzo a octubre 2015, se realizaron búsquedas de refugios diurnos mediante recorridos en lancha a lo largo de ríos, arroyos y lagunas, en once sitios del Área de Protección de Flora y Fauna Laguna de Términos, Campeche México. Por cada refugio localizado se registró el tamaño del grupo y su acomodo en el refugio, el tipo de refugio, el tipo de vegetación, la cobertura, la distancia entre refugios, el diámetro del tronco, la salinidad y el pH. Para investigar el efecto de las variables registradas en los refugios sobre el tamaño de cada grupo, se ajustaron Modelos Lineales Generalizados. Resultados: Encontramos 48 refugios en troncos secos, cortezas de árboles y puentes, asociados a cuerpos de agua dulce y oligohalina, principalmente en vegetación de manglar. El promedio ( \pm EE) del tamaño del grupo fue de $5.3 \pm 0.48$ para los refugios naturales y de $22.6 \pm 9.54$ para los artificiales. Encontramos una relación significativa con el tipo de refugio, el tipo de vegetación y el diámetro del tronco. Los refugios con tamaño de grupo mayores presentaron un acomodo agregado. Se localizaron 40 refugios sobre cuerpos de agua dulce y ocho en oligohalina con alto nivel de agrupamiento. Conclusiones: La mayor incidencia de refugios en sitios de agua dulce y ligeramente ácida, es explicada bajo la teoría del óptimo forrajeo. Debido a que el área de estudio se encuentra en el límite de su distribución, el manglar representa el tipo de vegetación más importante para garantizar la permanencia de las poblaciones de $R$. naso. Adicionalmente, debido a que en algunas porciones de la zona de estudio no fue posible encontrar refugios, es crucial llevar a cabo estudios sobre la calidad del agua y los niveles de contaminación en la región. Este estudio proporciona información relevante sobre las poblaciones del murciélago Rhynchonycteris naso con implicaciones directas sobre su conservación en zonas ribereñas y humedales costeros.

Palabras clave: Área de Protección de Flora y Fauna; ecología del refugio; especie amenazada; murciélago narigón; parámetros fisicoquímicos; salinidad; sitio de percha.

\section{REFERENCIAS}

Agraz, C.M., Osti, J., Chan, C., Arriaga, V., Acosta, J., Castillo, S., ... Martínez, J. (2015). Grado de conservación del ecosistema de mangle en la Laguna de Términos, Campeche: Propuesta de políticas ambientales y acciones de restauración. In J. Ramos \& G.J. Villalobos (Eds.), Aspectos socioambientales de la región de la Laguna de Términos, Campeche (pp. 117-132). Campeche, México: Universidad Autónoma de Campeche.

Al-Shami, S.A., Rawi, C.S.M., HassanAhmad, A., \& Nor, S.A.M. (2010). Distribution of Chironomidae (Insecta: Diptera) in polluted rivers of the Juru River Basin, Penang, Malaysia. Journal of Environmental Sciences, 22(11), 1718-1727.

Amezcua-Linares, F., \& Yáñez-Arancibia, A. (1980). Ecología de los sistemas fluvio-lagunares asociados a la Laguna de Términos. El hábitat y estructura de las comunidades de peces. Anales del Centro de Ciencias Del Mar y Limnología-UNAM, 7(1), 69-117.

Andriolo, A., Rodrigues, F.P., Zerbini, A., \& Barrio, J. (2010). Population estimates. In J.M. Duarte \& S. González (Eds.), Neotropical Cervidology: Biology and medicine of Latin American deer (pp. 271-282). Gland, Switzerland: FUNEP, Jaboticabal, Brazil \& IUCN.

Anon. (1958). The Venice system for the classification of marine waters according to salinity. Limnology and Oceanography, 3(3), 346-347. Retrieved from https:// aslopubs.onlinelibrary.wiley.com/doi/epdf/10.4319/ 10.1958.3.3.0346

Ávila-Flores, R., \& Medellín, R.A. (2004). Ecological, taxonomic, and physiological correlates of cave use by mexican bats. Journal of Mammalogy, 85(4), 675-687.

Biscardi, S., Russo, D., Casciani, V., Cesarini, D., Mei, M., \& Boitani, L. (2007). Foraging requirements of the endangered long-fingered bat: the influence of microhabitat structure, water quality and prey type. Journal of Zoology, 273(4), 372-381. 
Bradbury, J.W., \& Emmons, L.H. (1974). Social organization of some Trinidad bats: I. Emballonuridae. Zeitschrift Für Tierpsychologie, 36, 137-183.

Bradbury, J.W., \& Vehrencamp, S.L. (1976). Social organization and foraging in emballonurid bats: I. Field studies. Behavioral Ecology and Sociobiology, 1(4), 337-381.

Breviglieri, C.P.B., \& Uieda, W. (2014). Tree cavities used as diurnal roosts by Neotropical bats. Folia Zoologi$c a, 63(3), 206-215$.

Cain, D.J., Luoma, S.N., Carter, J.L., \& Fend, S.V. (1992). Aquatic insects as bioindicators of trace element contamination in cobble-bottom rivers and streams. Canadian Journal of Fisheries and Aquatic Sciences, 49(10), 2141-2154.

Caro, T. (2005). The adaptive significance of coloration in mammals. BioScience, 55(2), 125-136.

Ceballos, G., Blanco, S., González, C., \& Martínez, E. (2006). Rhynchonycteris naso (Murciélago) delimitada, con base al Atlas Mastozoológico de México. Distribución potencial. Retrieved from http://www. conabio.gob.mx/informacion/gis

Ceballos, G., \& Oliva, G. (2005). Los mamíferos silvestres de México. D.F., México: FCE, CONABIO.

Chambers, C.L., Alm, V., Siders, M.S., \& Rabe, M.J. (2002). Use of artificial roosts by forest-dwelling bats in Northern Arizona. Wildlife Society Bulletin, 30(4), 1085-1091.

Chi-Coyoc, T., Escalona-Segura, G., Vallarino-Moncada, A., Vargas-Contreras, J.A., Castillo-Vela, G.E., \& Lara-Reyna, J. (2016). Organochlorine and anticholinergic pesticides in wild mice from wetland ecosystems of the Gulf of Mexico. Therya, 7(3), 465-482.

Crawley, M.J. (2013). The R Book. Chichester, United Kingdom: John Wiley \& Sons.

Dalquest, W. (1957). Observations on the sharp-nosed bat Rhynchiscus naso (Maximilian). The Texas Journal of Science, 9, 219-226.

Davis, R., \& Cockrum, E.L. (1963). Bridges utilized as day-roosts by bats. Journal of Mammalogy, 44(3), 428-430.

de Conno, C., Nardone, V., Ancillotto, L., De Bonis, S., Guida, M., Jorge, I., ... Russo, D. (2018). Testing the performance of bats as indicators of riverine ecosystem quality. Ecological Indicators, 95, 741-750.

de la Lanza, G., Ramírez-García, P., Yves-F.T., \& Alcántara, A. (1993). La vegetación de manglar en la Laguna de Términos, Campeche, evaluación preliminar a través de imágenes LANDSAT. Hidrobiológica, 3, 1-2. de Sousa Barros, J., Bernard, E., \& Lopes Ferreira, R. (2020). Ecological preferences of neotropical cave bats in roost site selection and their implications for conservation. Basic and Applied Ecology, 45, 31-41.

Durack, P.J., Wijffels, S.E., \& Boyer, T.P. (2013). Longterm salinity changes and implications for the global water cycle. In G. Siedler, S. Griffies, J. Gould, \& J. Church (Eds.), Ocean Circulation and Climate A 21st Century Perspective (pp. 727-757). Oxford, United Kingdom: Academic Press.

Emmons, L.H., \& Feer, F. (1997). Neotropical rainforest mammals: a field guide. Chicago, United States: The University of Chicago Press.

Estrada-Villegas, S., Meyer, C.F.J., \& Kalko, E.K.V. (2010). Effects of tropical forest fragmentation on aerial insectivorous bats in a land-bridge island system. Biological Conservation, 143(3), 597-608.

Fenton, M.B., Bernard, E., Bouchard, S., Hollis, L., Johnston, D.S., Lausen, C.L., ... Zigouris, J. (2001). The bat fauna of Lamanai, Belize: roosts and trophic roles. Journal of Tropical Ecology, 17(4), 511-524.

Fernandes, M. (2000). Association of mammals with mangrove forest: a world wide review. Boletim Do Laboratório de Hidrobiologia, 13, 83-108.

Frith, C.B., \& Frith, D.W. (1985). Seasonality of insect abundance in an Australian upland tropical rainforest. Australian Journal of Ecology, 10(3), 237-248.

García, E. (2004). Modificaciones al sistema de clasificación climática de Köppen. México D.F., México: Instituto de Geografia-UNAM.

Gold-Bouchot, G., Noreña-Barroso, E., \& Zapata-Pérez, O. (1995). Hydrocarbon concentrations in the American oyster, Crassostrea virginica, in Laguna de Terminos, Campeche, Mexico. Bulletin of Environmental Contamination and Toxicology, 54(2), 222-227.

Goodwin, G.G., \& Greenhall, A.M. (1961). A review of the bats of Trinidad and Tobago. Bulletin of the American Museum of Natural History, 122(3), 187-302.

Günther, L., Lopez, M.D., Knörnschild, M., Reid, K., Nagy, M., \& Mayer, F. (2016). From resource to female defence: the impact of roosting ecology on a bat's mating strategy. Royal Society Open Science, 3,160503 .

Hogarth, P.J. (2007). The biology of mangroves and seagrasses. New York, United States: Oxford University Press.

INE-SEMARNAP. (1997). Programa de manejo del Área de Protección de Flora y Fauna "Laguna de Términos". México D.F., México: Instituto Nacional de Ecología. 
INEGI. (2010). Red hidrográfica. Mapa vectorial, escala 1:50000. INEGI.

INEGI. (2014). Mapa de uso de suelo y vegetación serie VI. Mapa vectorial, escala 1:250000. INEGI.

Infante-Mata, D., Moreno-Casasola, P., \& Madero-Vega, C. (2014). ¿Pachira aquatica, un indicador del límite del manglar? Revista Mexicana de Biodiversidad, 85(1), 143-160.

Jackson, J.K., \& Fisher, S.G. (1986). Secondary production, emergence, and export of aquatic insects of a Sonoran Desert stream. Ecology, 67(3), 629-638.

Jones, G., Jacobs, D., Kunz, T., Willig, M., \& Racey, P. (2009). Carpe noctem: the importance of bats as bioindicators. Endangered Species Research, 8, 93-115.

Kalcounis-Rueppell, M.C., Payne, V.H., Huff, S.R., \& Boyko, A.L. (2007). Effects of wastewater treatment plant effluent on bat foraging ecology in an urban stream system. Biological Conservation, 138, 120-130.

Kauffman, L., \& Rousseuw, P. (1990). Finding groups in data: an introduction to cluster analysis. New Jersey, United States: John Wiley \& Sons.

Kerth, G., \& König, B. (1999). Fission, fusion and nonrandom associations in female Bechstein's bats (Myotis bechsteinii). Behaviour, 136(9), 1187-1202.

Kerth, G., \& König, B. (2001). Day roost selection in female Bechtein's bats (Myotis bechteinii): a field experiment to determine the influence of roost temperature. Oecologia, 126, 1-9.

Knörnschild, M., Harview, C., Moseley, R., \& von Helversen, O. (2009). Remaining cryptic during motion-behavioral synchrony in the proboscis bat (Rhynchonycteris naso). Acta Chiropterologica, 11(1), 208-211.

Korine, C., Adams, A.M., Shamir, U., \& Gross, A. (2015) Effect of water quality on species richness and activity of desert-dwelling bats. Mammalian Biology, 80(3), 185-190.

Kunz, T.H. (1982). Roosting ecology of bats. In T.H. Kunz (Ed.), Ecology of bats (pp. 1-55). New York, United States: Plenum Publishing Corporation.

Kunz, T.H., \& Lumsden, L.F. (2003). Ecology of cavity and foliage roosting bats. In T.H. Kunz \& M.B. Fenton (Eds.), Bat ecology (pp. 3-89). Chicago, United States: The University of Chicago Press.

Laverty, T.M., \& Berger, J. (2020). Do bats seek clean water? A perspective on biodiversity from the Namib Desert. Biological Conservation, 248, 108686.

Lilley, T.M., Ruokolainen, L., Meierjohann, A., Kanerva, M., Stauffer, J., Laine, V.N., ... Nikinmaa, M. (2013).
Resistance to oxidative damage but not immunosuppression by organic tin compounds in natural populations of Daubenton's bats (Myotis daubentonii). Comparative Biochemistry and Physiology, Part C, 157, 298-305.

Lim, B., \& Miller, B. (2016). Rhynchonycteris naso. The IUCN red list of threatened species 2016: e.T19714A22010818. Retrieved from: https://www. iucnredlist.org/species/19714/22010818

Maechler, M., Rousseeuw, P., Struyf, A., Hubert, M., \& Hornik, K. (2019). Cluster: cluster analysis basics ands extensions (R Package Version 2.0.1). Retrieved from https://cran.r-project.org/web/packages/cluster/ cluster.pdf

McConville, A., Law, B.S., \& Mahony, M.J. (2013). Mangroves as maternity roosts for a colony of the rare east-coast free-tailed bat (Mormopterus norfolkensis) in South-eastern Australia. Wildlife Research, 40(4), 318-327.

Medellín, R.A., Arita, H.T., \& Sánchez, O. (2007). Identificación de los murciélagos de México: Clave de campo. D.F., México: Instituto de Ecología, UNAM.

Merilaita, S., Tuomi, J., \& Jormalainen, V. (1999). Optimization of cryptic coloration in heterogenous habitats. Biological Journal of the Linnean Society, 67(2), 151-161.

Mering, E., \& Chambers, C.L. (2014). Thinking outside the box: a review of artificial roosts for bats. Wildlife Society Bulletin, 38(4), 741-751.

Navarrete-Salgado, N.A., Fernández-Guillermo, E., \& Contreras-Rivero, G. (2004). Abundancia de Quironómidos (Diptera: Chironomidae) en el bordo "JC" del norte del Estado de México en el periodo de secas. Hidrobiológica, 14(2), 157-160.

Nogueira, M.R., \& Pol, A. (1998). Observações sobre os hábitos de Rhynchonycteris naso (Wied-Neuwied, 1820) e Noctilio albiventris Desmarest, 1818 (Mammallia, Chiroptera). Revista Brasileira de Biologia, 58(3), 473-480.

O’Farrell, M.J., \& Miller, B.W. (1997). A new examination of echolocation calls of some neotropical bats (Emballonuridae and Mormoopidae). Journal of Mammalogy, 78(3), 954-963.

Pacifici, M., Rondinini, C., Rhodes, J.R., Burbidge, A.A., Cristiano, A., Watson, J.E.M., ... Di Marco, M. (2020). Global correlates of range contractions and expansions in terrestrial mammals. Nature Coтmunications, 11, 1-9.

Plumpton, D.L., \& Jones, J.K. (1992). Rhynchonycteris naso. Mammalian Species, 413, 1-5.

R Core Team. (2017). R: A language and environment for statistical computing. R Foundation for Statistical 
Computing, Vienna, Austria. Retrieved from: http:// www.R-project.org/

Racey, P.A. (1998). The importance of the riparian environment as a habitat for British bats. In N. Dunstone \& M. Gorman (Eds.), Behaviour and Ecology of Riparian Mammals (pp. 69-92). Cambridge, United Kingdom: Cambridge University Press.

Ramsar. (2020). The list of wetlands of international importance. Retrieved from: https://www.ramsar.org/sites/ default/files/documents/library/sitelist.pdf

Razgour, O., Korine, C., \& Saltz, D. (2010). Pond characteristics as determinants of species diversity and community composition in desert bats. Animal Conservation, 13(5), 505-513.

Reid, F. (1997). A field guide to the mammals of Central America and Southeast Mexico. New York, United States: Oxford University Press.

Rodríguez-Durán, A. (2003). Bat assemblages in the west Indies: the role of caves. In T.H. Fleming \& P.A. Racey (Eds.), Island Bats: Evolution, Ecology, and Conservation (pp. 265-280). Chicago, United States: The University of Chicago Press.

Russ, J.M., \& Montgomery, W.I. (2002). Habitat associations of bats in Northern Ireland: implications for conservation. Biological Conservation, 108, 49-58.

Salas, J.A. (2010). Diversidad y ecología de los quirópteros como indicadores del estado de conservación de la reserva de producción de fauna "Manglares el Salado” (Tesis de Maestría). Universidad de Guayaquil, Ecuador.

Salas, J.A., Viteri, F.H., Zambrano, M.M., Benavides, V.H., \& Carvajal, R.M. (2013). Distribution extension of Proboscis bat Rhynchonycteris naso (Wied-Neuwied, 1820) (Chiroptera: Emballonuridae): new record for Southwestern Ecuador. Check List, 9(5), 1054-1056.

Santana, S.E., Dial, T.O., Eiting, T.P., \& Alfaro, M.E. (2011). Roosting ecology and the evolution of pelage markings in bats. PLOS ONE, 6(10), e25845.

SEMARNAP. (2000). Programa de manejo Reserva de la Biosfera Pantanos de Centla. México D.F., México: Instituto Nacional de Ecología.

SEMARNAT. (2010). Norma Oficial Mexicana NOM059-SEMARNAT-2010. Protección ambiental-Especies nativas de México de flora y fauna silvestres-Categorías de riesgo y especificaciones para su inclusión, exclusión o cambio-Lista de especies en riesgo. Diario Oficial de La Federación $(D O F)$. Retrieved from https://biblioteca.semarnat. gob.mx/janium/Documentos/Ciga/agenda/DOFsr/ DO2454.pdf

Simmons, N.B., \& Voss, R.S. (1998). The mammals of Paracou, French Guiana: a Neotropical lowland rainforest fauna. Part 1. Bats. Bulletin of the American Museum of Natural History, 237, 1-219.

Soares, F.A.M., Graciolli, G., Ribeiro, C.E.B.P., Bandeira, R.S., Moreno, J.A.T., \& Ferrari, S.F. (2016). Bat (Mammalia: Chiroptera) diversity in an area of mangrove forest in Southern Pernambuco, Brazil, with a new species record and notes on ectoparasites (Diptera: Streblidae). Papéis Avulsos de Zoologia, 56(6), 63-68.

Stahlschmidt, P., \& Brühl, C.A. (2012). Bats at risk? Bat activity and insecticide residue analysis of food items in an apple orchard. Environmental Toxicology and Chemistry, 31(7), 1556-1563.

Stephens, D.W., \& Krebs, J.R. (1986). Foraging theory. New Jersey, United States: Princeton University Press.

Uc-Cua, G.M. (2017). Caracterización de sitios de percha del murciélago Rhynchonycteris naso en la Reserva de la Biosfera Pantanos de Centla (Tesis de Maestría). El Colegio de la Frontera Sur, México.

Vannucci, M. (2001). What is so special about mangroves? Brazilian Journal of Biology, 61(4), 599-603.

Vaughan, N., Jones, G., \& Harris, S. (1996). Effects of sewage effluent on the activity of bats (Chiroptera: Vespertilionidae) foraging along rivers. Biological Conservation, 78(3), 337-343.

Vebrová, L., Nieuwenhuijzen, A., Kolář, V., \& Boukal, D.S. (2018). Seasonality and weather conditions jointly drive flight activity patterns of aquatic and terrestrial chironomids. BMC Ecology, 18(1), 19.

Venables, W.N., \& Ripley, B.D. (2002). Modern applied statistics with $S$ ( $4^{\text {th }}$ Edition). New York, United States: Springer.

Villa-R.B. (1966). Los murciélagos de México. D.F. México: Instituto de Biología, Universidad Nacional Autónoma de México.

Waldbauer, G. (2006). A walk around the pond. Cambridge, United States: Harvard University Press.

Whitehurst, I.T., \& Lindsey, B.I. (1990). The impact of organic enrichment on the benthic macroinvertebrate communities of a lowland river. Water Research, 24(5), 625-630.

Wickham, H. (2016). ggplot2: Elegant graphics for data analysis. New York, United States: Springer-Verlag.

Wilde, L.R., Günther, L., Mayer, F., Knörnschild, M., \& Nagy, M. (2018). Thermoregulatory requirements shape mating opportunities of male Proboscis bats. Frontiers in Ecology and Evolution, 6, 199.

Williams, W.D., Boulton, A.J., \& Taaffe, R.G. (1990). Salinity as a determinant of salt lake fauna: a question of scale. Hydrobiologia, 197(1), 257-266. 
Willis, C.K.R., \& Brigham, R.M. (2004). Roost switching, roost sharing and social cohesion: forest-dwelling big brown bats, Eptesicus fuscus, conform to the fissionfusion model. Animal Behaviour, 68(3), 495-505.
Yáñez-Arancibia, A., \& Day, J.W. (1982). Ecological characterization of Terminos Lagoon, a tropical lagoonestuarine system in the Southern Gulf of Mexico. Acta Oceanologica, 5(4), 431-440. 